\title{
Guidelines of the Italian Society of Videosurgery in Infancy for the minimally invasive treatment of pediatric nephrectomy and partial nephrectomy
}

\author{
Salvatore Fabio Chiarenza, ${ }^{1}$ Cosimo Bleve, ${ }^{1}$ Ciro Esposito, ${ }^{2}$ Maria Escolino, ${ }^{2}$ Fabio Beretta, ${ }^{3}$ \\ Maurizio Cheli, ${ }^{4}$ Vincenzo Di Benedetto, 5 Maria Grazia Scuderi, 5 Giovanni Casadio, 6 Maurizio Marzaro, ${ }^{7}$ \\ Leon Francesco Fascetti,8 Pietro Bagolan,9 Claudio Vella, 10 Maria Luisa Conighi,1 Daniela Codric, 11 \\ Simona Nappo, 12 Paolo Caione 13 \\ 1Pediatric Surgery and Urologic Unit, Regional Center of Minimally Invasive Surgery and Urology, S. Bortolo Hospital, \\ Vicenza; ${ }^{2}$ Pediatric Surgery Unit, Federico II University, Naples; ${ }^{3}$ Pediatric Surgery Unit, APSS, Trento; 4 Pediatric Surgery \\ Unit, Papa Giovanni XXIII Hospital, Bergamo; ${ }^{5}$ Pediatric Surgery Unit, Vittorio Emanuele Hospital, Catania; ${ }^{6}$ Pediatric \\ Surgery Unit, Parma University Hospital, Parma; ${ }^{7}$ Pediatric Surgery Unit, Local Health Unit 2, Treviso Hospital; ${ }^{8}$ Pediatric \\ Surgery Unit, Padua University; ${ }^{9}$ Neonatal Pediatric Surgery, Pediatric Hospital Bambino Gesù, Rome; ${ }^{10}$ Pediatric Surgery \\ Unit, Children Hospital V. Buzzi, Milan; ${ }^{11}$ Pediatric Surgery Unit, IRCCS Burlo Garofolo, Trieste; 12 Pediatric Surgery Unit \\ Regina Margherita Hospital, Turin; ${ }^{13}$ Pediatric Surgery and Urologic Unit, Pediatric Hospital Bambino Gesù, Rome, Italy
}

\section{Background}

The SIVI (Italian Society of Videosurgery in Infancy) guidelines are clinical practice guidelines edited and approved by the Society's steering committee. They are the products of a detailed systematic review of the literature, integrated with expert opinion in the field of pediatric minimally invasive surgery.

These guidelines are intended to assist the pediatric surgeons experienced or not experienced in minimally invasive surgery, with the goal to inform choices related to the indications, approach, and techniques to use when treating the major pediatric surgical pathologies.

Given the complexities of congenital malformations and other pediatric surgical conditions, as well as large variations in available regional health services, we must note that these guidelines are not intended as a cookbook recipe to follow for all possible patients. Rather, the guidelines should serve as a flexible framework, to be used by the physician in concert with the parents, to choose the best approach for each individual patient. Decisions tailored to available scientific knowledge and the needs and

Correspondence: Salvatore Fabio Chiarenza, Pediatric Surgery and Urologic Unit, Regional Center of Minimally Invasive Surgery and Urology, S. Bortolo Hospital, viale Rodolfi 37, 36100, Vicenza, Italy. Tel.: +39.0444.752642.

Fax: +39.0444 .752643$

E-mail: fabio.chiarenza@aulss8.veneto.it

Key words: SIVI; minimally invasive treatment, pediatric nephrectomy; pediatric partial nephrectomy.

Received for publication: 13 December 2019.

Accepted for publication: 16 December 2019.

This work is licensed under a Creative Commons Attribution NonCommercial 4.0 License (CC BY-NC 4.0).

OCopyright: the Author(s), 2019

Licensee PAGEPress, Italy

La Pediatria Medica e Chirurgica 2019; 41:231

doi:10.4081/pmc.2019.231 desires of the patient's family serve both patient autonomy and medical science.

All guidelines are published in this scientific Journal, in order to ensure their availability to all physicians.

The Guidelines project has been approved by the SIVI General Assembly of the 2016 Madrid congress.

Review of guidelines has been performed by the Steering Committee of SIVI and experts.

\section{Introduction}

Throughout history, the pediatric laparoscopic nephrectomy was first described at the beginning of the Nineties by Erlich and colleagues in a child and by Koyle and colleagues in an unweaned patient. In both cases, the patients were suffering from multicystic dysplastic kidney. Since then, the general principles of the transperitoneal laparoscopic nephrectomy have remained substantially unvaried. Initially, the large-caliber trocars and the excessive length of the instruments made this procedure difficult to be performed in younger patients, however the progressive instrument miniaturization allowed a rapid adoption and diffusion of this technique. It is important to take into account that the laparoscopic procedure in young patients is different to the one performed on adults, not only for the type of instruments used, but also for the anatomy and physiology of the young patient. The relatively short distance between the access points and the intra-abdominal organs could potentially lead more easily to accidental lesions of the organs, and the pneumoperitoneum could have greater effects on the respiratory, cardiac and renal physiology. Every surgeon that would like to undertake the challenge of this type of surgery needs a clear understanding of the surgical technique, the instruments and the physiology of the child.

\section{Laparoscopic nephrectomy}

\section{Indications for surgery}

The indications for the nephrectomy are represented by the congenital or acquired conditions that implicate the presence of a 
nonfunctional and/or damaging kidney, and that are not treatable with conservative therapies. Among them, we can mention: cystic dysplastic kidney (or multicystic kidney); nonfunctioning dysplastic kidney associated with vesicoureteral reflux; functioning silent hydronephrotic kidney/infected kidney; kidney-mediated hypertension not responding to pharmacological therapy; congenital nephrotic syndrome with protein loss. The Multicystic Dysplastic Kidney (MCDK) is characterized by the presence of multiple cyst lesions that resembles a bunch of grapes, with various positions and localizations, inside a variably scarce renal parenchyma. This condition has an incidence that varies between 1:3100-1:4300 live births and it occurs mainly in males, affecting mainly the left kidney. The condition occurs bilaterally in $19 \%$ to $43 \%$ of cases and it is incompatible with life (associated in uterus with anhydramnios and pulmonary dysplasia). From a histological perspective, the MCDK presents an abnormal metanephrinic differentiation that reveals the presence of primitive ducts, metaplastic cartilage, ductules and primitive glomerules with associated normal nephrogenic elements. The etiology and pathogenesis are not distinctive: there are evidences supporting the obstructive origin of the condition (the precocious fetal ureteral obstruction leads to renal dysplasia) or the genetic origin (abnormal expression of PAX2 and BCL2). The majority of MCDK cases are nowadays diagnosed in utero and are asymptomatic. The postnatal manifestation of the condition includes abdominal mass, lumbar pain, urinary tract infections, hypertension. Abnormal contralateral urinary conditions are found in $20-40 \%$ of cases, the most frequent is represented by the vesicoureteral reflux. The diagnostic confirmation and the postnatal workup are based upon: ultrasound monitoring of the urinary system to document the kidney involution; a Tc99-DMSA renal scintigraphy that demonstrates absent function for the dysplastic kidney; a voiding cystourethrogram is recommended in case there is a suspect of anatomical abnormalities for the contralateral kidney (grade D) or dilated retrovesical ureter. The MCDK treatment is normally conservative, supported by a high percentage of spontaneous regression (documented in over $60 \%$ of cases, half of them within 24 months after birth) and by a low risk to develop hypertension or other malignancies. The systematic surgical ablation is not recommended however it might be performed in selected cases after adequate consent from the patient's parents. Surgery might be recommended in case of voluminous renal mass or increased dimension, coexistence of abnormalities in the renal-ureteral axis as the ectopic ureteral outlet associated with MCDK. The hypertension might be another condition for which the surgical ablation is indicated. Nowadays, the preferred approach should be the minimally-invasive one, either transperitoneal (laparoscopic) or retroperitoneal. The open nephrectomy, which represented the gold standard until one or two decades ago, usually has a limited application (ex. newborns, low-weight children with severe complex multiple malformations or in cases when the minimally invasive technique cannot be performed).

\section{Preoperative work-up}

The blood exams that are normally required are: complete blood count, creatinine, electrolytes, coagulation tests and, eventually, blood type. Especially for the transperitoneal laparoscopic procedures, it is recommended to perform a bowel preparation with antifermenting drugs and laxatives and/or an enema 24 hours before surgery. The surgery consent form should include information on the diagnosis, the recommended surgical procedure (preferred minimally invasive access), the surgical alternatives and the possible need of conversion to laparotomy. It is recommended to inform on the type of stent and catheters that will be used and ultimately on the possible complications, in particular bleeding.

\section{Operating room preparation}

In the transperitoneal laparoscopic approach, the patient is normally positioned on a lateral or semi-lateral position with the flank opposed to the interested one positioned towards the edge of the surgical table to facilitate the movement of the surgical instruments. The operating surgeon stands in front of the patient, with the laparoscopic video tower positioned across the table from the surgeon. The 3- or 5-mm laparoscopic instruments normally include (according to the operator's habit and/or preference): 1-2 needle holders, swab, graspers, coagulation hook, mixters, scissors, monopolar and/or bipolar electrosurgical devices, aspirator-irrigator. The use of laparoscopic fixation and dissection devices that make use of different power sources (LigaSure, Starion, Ultracision, advanced monopolar and bipolar devices) normally allows a reduction in the procedure length; these devices also need to be prepared according to the preference and choice of the operating surgeon. It is recommended to make fixation devices as clips and/or EndoGIA available for the laparoscopic vessels closure. In case of retroperitoneoscopic approach with lateral access, the patient is positioned on a lateral decubitus with a soft pillow, or roll, under the contralateral flank in order to widen the space between the costal margin and the iliac crest allowing the positioning of the laparoscopic ports. The operating surgeon and the assistant stand behind the patient, the surgical nurse stands next to them or at the feet and the laparoscopic video tower is positioned on the other side, i.e. towards the abdomen. The laparoscopic instruments are analogous to the ones used in the transperitoneal access. In case of minimally invasive posterior retroperitoneal access, the patient is arranged on a prone position, with chest and hips lifted by suitable pillows in order to obtain the decompression of the abdomen (see specific section). It is always appropriate to have at disposal a set of surgical instruments for open surgery, to be used in case of possible conversion to open surgery.

\section{Surgical technique}

The pediatric nephroureterectomy and the partial nephroureterectomy can be performed via: 1) transperitoneal approach; 2) retroperitoneal approach, this latter being performed with lateral or posterior approach. Following, the peculiarities of the video-laparoscopic procedures for the partial nephroureterectomy surgery are presented singularly.

\section{Transperitoneal laparoscopic nephrectomy}

\section{The patient position}

The correct patient positioning on the operating table is the first critical element in performing the laparoscopic nephrectomy and it has to be performed jointly by the surgeon, the anesthesiologist and the nursing staff in order to prevent possible problems related to the organ exposition and the patient monitoring and safety. The patient position might vary from the complete to the partial 30-degree lateral decubitus according to the surgeon's preference and the patient size and body weight; the diameter and the location of the trocars vary according to the ergonomics, the kidney dimension, and certainly the young patient size. Independently from the position, it might be useful to break the operating table to widen the space between the costal margin and the hip in order to efficaciously increase the working space. In younger patients, the break of the operating table might not provide any benefit. In case the patient is positioned on a lateral decubitus, it is important to carefully position the arms and legs to avoid joint tractions and possible pressure sores.

Once positioned, the patient is then secured to the operating 
table to allow, if necessary, table rotations or deflections (Trendelemburg/Antitrendelemburg position). It is important to secure legs and arms, pelvis, chest, shoulders and head. Once the patient is positioned, the surgeons stand in front of him/her, with the laparoscopic tower and the monitor are positioned across the table from the surgeon. The surgical nurse stands, together with the surgical instrument trolley, to the right of the surgeon.

\section{Access to the peritoneal cavity and positioning of the 1st trocar}

When performing the pediatric transperitoneal laparoscopic nephrectomy, it is recommended to perform an open access to the peritoneum with a skin incision at the umbilical level (trans-, supra- or subumbilical incision). Once the peritoneum is opened, the $1^{\text {st }}$ trocar is inserted under direct vision. After confirming the correct positioning of the trocar, the pneumoperitoneum is established (pressure of 6-12 $\mathrm{mmHg}$ according to the patient's age and weight). The abdominal cavity is then explored with a $5-\mathrm{mm}$ or $10-$ mm possibly 30-degree optical trocar; according to the surgeon's preference, a 0 -degree optical trocar might be used.

\section{Surgical trocars placement}

The number and especially the location of the trocars has to be carefully selected by the surgeon in relation to the preferred technique and to the laparoscopic instruments in use. Two working trocars are placed under direct vision, ideally along the lateral margin of the ipsilateral rectus muscle, respecting, as much as possible, the surgical instruments triangulation. In case of retraction of the liver and spleen, a fourth trocar might be positioned on the midline, below the xiphoid process or in the contralateral hypochondrium, according to the operating surgeon's discretion.

\section{Details of transperitoneal video-laparoscopic technique}

The mobilization and dissection of the colon along the white line of Toldt from the splenic flexure (left side) or the hepatic flexure (right side) is performed. Once the ureter is identified, running anteriorly to the psoas muscle, it has to be followed to the renal hilum (it can be used for traction). The renal hilum is carefully identified, and the hilum vessels are isolated bluntly (the renal artery and the renal vein are to be isolated separately). Once the artery (first) and the vein (after) are isolated, these are sealed with different possible methods according to the surgeon's preference and the availability of the instruments in the operating room; it is therefore possible to use ligations, endoclips or other fixation devices with radiofrequency or ultrasound energy (LigaSure, Starion, Ultracision, advanced bipolar device, etc.). Attention needs to be paid in identifying and selecting possible accessory renal veins (rather frequent) and, if necessary, the inferior adrenal veins and the gonadal veins. The kidney is then isolated, and the superior and lateral ligaments are dissected. The ureter is dissected and ligated or clipped as distally as possible especially if refluxing; however, if the ureter is not refluxing for certain, it can be left open to favor the coaptation. In some circumstances, for example in case of markedly hydronephrotic kidney, the hilar vessels can be frayed in multiple small caliber collaterals that run on the surface of the pelvis that appears markedly distended: in this case it is important to pay great attention to their isolation and ligation. In case of small or dilated kidneys, removed for non-oncological reasons, the surgical specimen might be removed through the umbilical port, even without the use of an endobag. If necessary, the fascial incision can be extended, to allow the removal of the specimen. If the specimen is big in dimension or needs to be removed entirely, it might be necessary to use an endobag: the kidney is then removed through the umbilical port or through a counter incision in the lower abdominal quadrants (Pfannenstiel incision). The renal fossa has to be carefully inspected for hemostasis. The positioning of a drainage is elective according to the surgeon's decision. The accessory trocars are removed under direct vision and the umbilicus is easily sutured, normally with interrupted sutures. The accessory trocars incisions are sutured according to the surgeon's preference.

\section{Post-operative management}

The postoperative hospital stay is normally reduced to the minimum extent necessary. The discharge is normally possible two or three days after surgery according to the patient's general conditions and the absence of complications.

\section{Comments}

The transperitoneal access presents several advantages and disadvantages compared to the retroperitoneal access. Advantages: wide working space, especially in younger patients; preexisting space; easy access to the distal ureter with possible nearly complete excision of the ureter to the bladder. Disadvantages: slightly longer preoperative fasting; risk of accidental lesions to the intraabdominal organs; increased interferences with the respiratory and cardiac physiology; the procedure is not recommended in patient undergoing peritoneal dialysis.

\section{Retroperitoneoscopic nephrectomy}

The retroperitoneoscopic nephrectomy can be performed via two different access: lateral and posterior.

\section{Lateral approach}

The pediatric retroperitoneoscopic nephrectomy in a lateral position was first described in 1995 by Diamond and colleagues. Since then, it gained popularity even though, at the present moment, not every health care facility has sufficient experience with this particular access.

\section{Patient positioning}

First of all, a vesical catheter is placed. The patient is then positioned in a lateral decubitus position with break of the operating table and positioning of a roll under the declive flank to widen the space between the $12^{\text {th }}$ rib and the iliac crest. It is important that the patient's back is positioned towards the lateral edge of the operating table to allow a wide instrument angulation, avoiding the collision with the table edge. The operating surgeon and the assistant are both positioned facing the patient's back, the monitor is on the other side of the table, the scrub nurse is right to the operating surgeon.

\section{Surgical access}

The $12^{\text {th }}$ and $11^{\text {th }}$ rib, together with the iliac crest and the paraspinalis muscle, are identified. The anatomical landmarks can be marked with a dermographic pencil. A 1-cm incision is performed at the anterior margin of the $12^{\text {th }}$ rib. Once the splitting of the lateral abdominal muscles is completed, the posterior fascia is opened and it is possible to access to the retroperitoneum (presence of retroperitoneal fat). The blunt dissection and the creation of the retroperitoneal space are performed with moist gauzes or with the only aid of the optic ( 0 - or 30-degree) and the gas insufflation pressure. The space is created with blunt dissection with a moist gauze, digital dissection, or with oscillating movement of the camera 
along the psoas muscle; due to the dissection and the introduction of $\mathrm{Co} 2$, the peritoneum moves anteriorly and superiorly, leaving the necessary space to insert the other working trocars. This maneuver has to be extremely delicate to avoid accidental lesions to the peritoneum that would allow the passage of gas in the abdomen, consequently losing the retroperitoneal space.

\section{Technique details}

Once the working space is created, two accessory 3- or 5-mm are positioned under vision, normally the first at the costovertebral angle, the other at the head of the anterosuperior iliac spine along the axillary line (after verifying the correct anterior mobilization of the peritoneum). Once the trocars are positioned, the main anatomical landmark is represented by the psoas muscle that lies horizontally and inferiorly to the operating field. The kidney is suspended upwards on the peritoneum and it is approached posteriorly, identifying the hilum and the hilar vessels. During the first phase, it is important that the kidney is not detached anteriorly and superiorly from the peritoneum and the diaphragm: the pressure of insufflation gently pushes it forward and upward together with the peritoneum, allowing the visualization and dissection of the hilum. It might happen that, to allow the visualization of the renal hilum, the kidney might be constantly lifted with one of the two instruments used by the surgeon, forcing him/her to perform the dissection of the hilum with only one instrument; in this case it might be necessary to place a third 3-mm service trocar to keep the kidney lifted. The retroperitoneoscopic access allows an easier identification of the vessels and the correct ligation of the artery first and the vein after. The ureter is then dissected (see method on the transperitoneal nephrectomy section). Finally, the kidney is isolated at the poles and from the anterior peritoneal surface, obtaining a complete mobilization. The surgical specimen is removed through the optic trocar access, widening the incision if needed.

\section{Comments}

Even though the retroperitoneoscopic approach allows an easy access to the renal vessels at the hilum, the space creation in the retroperitoneum and in renal fossa, together with the correct understanding of the anatomy, requires high skills and specific training. In order to maintain the orientation, the psoas muscle always has to be identified and visualized at the base of the operating field, whereas the kidney has to be located in the anterosuperior part of the field. The great vessels (inferior vena cava on the right and aorta on the left) are relatively close and have to be carefully respected to avoid harmful lesions during the maneuvers in narrow spaces. Compared to the transperitoneal access, the cardiac and respiratory effects of the retropneumoperitoneum are unremarkable, and the risk of lesions to the intra-abdominal organs or the risk to develop adhesions is reduced to the minimum. The retroperitoneal access does not jeopardize the conversion to the open surgery with lateral access (normally preferred by the pediatric urology specialists).

\section{Prone access}

Described by Borzi, this approach has found limited diffusion. However, thanks to its particular characteristics, it is an appropriate approach in specific indications.

\section{Patient position and access}

After the insertion of a vesical catheter, the patient is placed in a prone position, with the chest and the pelvis lifted by pillows to avoid the compression of the abdomen allowing a wide distention and excursion of the abdominal wall: in this position, the intraabdominal organs distance from the retroperitoneum and fall for- ward. The young patient needs to be positioned towards the edge of the operating table, on the side that needs to undergo surgery.

\section{Surgical access}

The first access is performed at the apex of the $12^{\text {th }}$ rib with open technique. As in the lateral position access, the retroperitoneum is reached after splitting of oblique muscles and the space is created bluntly with a moist gauze or with the Gaur balloon technique.

\section{Technique details}

The second trocar (normally a 3- or 5-mm trocar) is positioned along the posterior axillary line (monitoring, under guidance, the correct anterior mobilization of the peritoneum). The third trocar (3or $5-\mathrm{mm}$ trocar as well) is positioned at the costovertebral angle, at the border of the paraspinal muscles. In this position, the psoas muscle is barely identifiable and it cannot be used as anatomical landmark. It is important to underline that, with the prone laparoscopic retroperitoneal access, the surgeon will observe the paraspinal muscles superiorly and the renal fossa inferiorly, opposite to what happens with the lateral approach, however the kidney vertically falls by gravity, facilitating the identification and the dissection of the hilum with no traction on the kidney itself. Moreover, an accidental opening of the peritoneum, that is more difficult to occur, does not represent a problem. The hilar vessels are easily identified, singularly isolated and ligated with clips or other devices, as described for the transperitoneal or retroperitoneal laparoscopic nephrectomy via lateral access. Finally, if the ureter is not refluxing, it can be left open or it can be ligated or closed with clips and then dissected. Once the isolation of the kidney is completed, the specimen is removed through the main trocar incision.

\section{Comments}

The posterior retroperitoneal laparoscopic access with prone patient is particularly useful in case of bilateral nephrectomies, for example in preparation to kidney transplant, in case of nephritic syndrome with severe proteinuria, dysplastic kidneys with severe hypertension or any other indication for bilateral nephrectomy. The main advantage consists in the possibility to perform a bilateral surgery without moving the patient and without violating the peritoneal cavity; this latter represents a fundamental element in pediatric patients undergoing peritoneal dialysis. Moreover, compared to the lateral access, the posterior access decreases the risk of causing peritoneal lacerations and the vascular pedicle is better exposed by the kidney falling forward. The disadvantage of the prone retroperitoneal access is that it requires particular endoscopic direction skill and an accurate study of the trocar positioning. For these reasons, it is a less familiar access for the pediatric urology surgeon.

\section{Partial nephroureterectomy}

\section{Indications for surgery}

The upper pole (more frequent) or lower pole (rarer) partial nephroureterectomy are indicated in pediatric patients with duplicated collecting system complicated by hydroureteronephrosis with dysplastic non-functioning system or as a consequence of sever nephropathy due to vesicoureteral reflux.

\section{Surgical technique}

The surgical technique is similar to the one used for the simple nephrectomy in its transperitoneal and retroperitoneal variations, with both lateral and posterior access. However, it is important to 
underlie some peculiarities. In order to make the procedure safer, as a precaution it is possible to preoperatively position an ascending catheter in the ureter of the moiety to be preserved; a cystoscopy is performed with the patient in lithotomy position and a ureteral catheter is positioned, possibly a 4- or 5-Ch Pollack catheter. As a precaution, the ureteral catheter can be anchored to the Foley vesical catheter until the end of the procedure. The laparoscopic time depends on the selected approach: transperitoneal or retroperitoneal.

\section{Transperitoneal approach}

The procedure is performed via transmesocolic access on the left and retromesocolic access on the right. After accessing the renal fossa, the two ureters are identified (note that the ureter to be preserved is catheterized). It might be useful to dissect the ureter moiety to be forgone $2-3 \mathrm{~cm}$ from the lower kidney pole to facilitate the exposition of the renal pole through its traction. The main vascular pedicle and the one of moiety that needs to be removed are identified; the vessels of the interested system are dissected (see nephrectomy). The pathological parenchyma is dissected following as much as possible the cleavage lines (using devices with ultrasound energy, monopolar or bipolar electrical energy, radiofrequency, according to the operator's preference). The integrity of the pelvicalyceal system of the residual system is verified injecting 3-4 $\mathrm{ml}$ of dye (dilated patent blue) through the already positioned ureteral catheter. In case of accidental opening of a calyx, this latter is sutured with absorbable sutures. The distal ureter is removed paying particular attention to avoid damages to the vascularization of the moiety ureter, starting the dissection along the muscle wall of the ureter that has to be removed. The ureter is ligated distally with ligature sutures or clips (if the ureter is not refluxing, it can be left open) and dissected as in the simple nephrectomy. The cut surface hemostasis is carefully verified on the parenchymal section, with possible application of fibrin glue or other sealing material. The drainage (preferably aspiration) is positioned in the renal fossa, outpouring from the most slanting laparoscopic port.

\section{Lateral or posterior retroperitoneal approach}

This approach retraces the stages of the already described retroperitoneoscopic simple nephrectomy, taking into account the specific instructions for the transperitoneal laparoscopic partial nephrectomy. The retroperitoneoscopic approach is less invasive compared to the transperitoneal one, with good patient tolerance, however, it can sometimes present the disadvantage of a more difficult distal dissection of the ureter in the pelvic cavity.

\section{Other surgical techniques}

\section{Single site nephrectomy}

This approach consists in a video-laparoscopic nephrectomy that uses only a single laparoscopic port, normally umbilical, wider than usual, with a specific trocar through which an optic and one or two working ports for the instruments are inserted. The approach is transperitoneal and it retraces the same stages previously described stages. This technique proves to be appropriate especially for young adults or school-age patients. Its advantage consists in the use of the umbilical scar only, avoiding small unaesthetic scars produced by the accessory trocars. The disadvantages consist in the use of specifically made instruments, moreover the technique requires significant training and experience and, above all, the dissection of the organ is more difficult (longer operative time). The assistance of a robot might improve the operating surgeon dexterity.

\section{Robot-assisted nephrectomy}

The use of the Da Vinci robot easies the work of the surgeon, however it does not modify the indications and the surgical stages for the above described nephrectomy. Even though it might be used also retroperitoneoscopically, the transperitoneal access is the preferred one as it offers a wider working space compared to the retroperitoneum. In spite of the high costs of using a robot, the availability of 5-mm instruments might probably widen the use of this device in pediatric patients, even though there are particular limitations in smaller patients (newborns or infants). The surgical robot is mainly used for the deconstructive video-laparoscopic surgery however its advantages are evident especially in the reconstructive surgery (ex. pyeloplasty). Due to the high management costs, its application is currently limited to the centers where it can be used by different surgical disciplines (general surgery, urology, gynecology, cardiac surgery, etc.).

\section{Laparoscopic ligation of the ureter}

Patients candidate for nephrectomy in preparation to kidney transplant were recommended to undergo a simplified videolaparoscopic surgery for ligation of the native ureter without removing the correspondent kidney. After ligation of the ureter, the kidney incurs to progressive involution and parenchymal fibrosis with glomerular atrophy, demonstrated at the histology exam. The procedure can be performed via laparoscopic transperitoneal or retroperitoneal approach and it retraces the same stages of the nephrectomy, however it presents an easier execution and a significantly reduced invasiveness compared to the nephrectomy. However, if the parenchymal atrophy does not occur rapidly, there is the risk to develop a hydronephrotic symptomatic sac.

\section{Post-operative management and follow-up}

The post-operative management $[\ldots]$ as the minimally invasive video-laparoscopic approach has reduced to the minimum the postoperative assistance and the hospital stay. The follow-up time varies according to the underlying condition of the young patient and the residual renal function. The respect for the residual renal function is essential and the monitoring has to continue until the patient reaches the full development; it is also important to periodically monitor the blood pressure. A different path is established for the patients that undergo the nephrectomy in preparation to kidney transplant: they are listed in the dialysis-transplant waiting list.

\section{Conclusions}

Nowadays the minimally invasive video-laparoscopic techniques have replaced the traditional open surgery in most of the cases that need to undergo deconstructive surgery. This is valid especially for the simple nephrectomy and the partial nephroureterectomy for benign conditions, whereas the indication for laparoscopic nephroureterectomy for oncological conditions (normally Wilm's tumor) is still under discussion. However, in particular clinical conditions (low-weight or premature babies, particular clinical conditions, coagulopathy, severe cardiac disease, etc.) the open approach is indicated and might be preferred by the urology surgeon. The choice between the transperitoneal and retroperitoneal laparoscopic approach depends mainly on the preference and experience of the pediatric urology surgeon, however it might 
sometimes be conditioned by specific patient's conditions (i.e.: retroperitoneal access in patient undergoing peritoneal dialysis; transperitoneal access for partial nephroureterectomy with ectopic distal ureter). The technological improvement of the available instruments has allowed a greater number of healthcare facilities to access to video-laparoscopic techniques to perform the nephrectomy and the partial nephroureterectomy; however, it is important to notice that, in order to safely use the minimally invasive techniques, an appropriate expertise is required from the surgeon and his/her team, combining patience, precision, perseverance and most of all prudence.

\section{References}

1. Ehrlich RM, Gershman A, Fuchs G. Laparoscopic renal surgery in children. J Urol 1994;151:735.

2. Jordan GH, Winslow BH. Laparoendoscopic upper pole partial nephrectomy with ureterectomy. J Urol 1993;150:940-3.

3. Al-Khaldi N, Watson AR, Zuccollo J, et al. Outcome of antenatally detected cystic dysplastic kidney disease. Arch Dis Child 1994;70:520.

4. Winyard PG, Risdon RA, Sams VR, et al. The PAX2 transcription factor is espressed in cystic and hyperproliferative dysplastic epithelia in human kidney malformation. J Clin Invest 1996;98:451.

5. NICE. Laparoscopic nephrectomy (including nephroureterectomy). Disponibile al sito: https://www.nice.org.uk/guidance/ ipg 136

6. Narchi H. Risk of hypertension with multicystic kidney disease:a systematic revew. ArchDis Child 2005;90:921.

7. Basant K, Vijai Datta U, Manish KG, et al. Early nphrectomy in unilateral multicystic dysplastic kidney in children cures hypertension early: an observation. Eur J Pediatr Surg 2017 (in press).

8. Scott RH, Walker L, Olsen OE, et al. Surveillance for Wilms tumor inat-risk children: pragmatic reccommendations for best practice. Arch Dis Child 2006;91:995.

9. Narchi H. Risk of Wilms' tumor with multicystic kidney disease: asystematic review. Arch Dis Child 2005;90:147.

10. Esposito C, Escolino M, Corcione F, et al. Twenty-year experience with laparoscopic and retroperitoneoscopic nephrectomy in children: considerations and details of technique. Surg Endoc 2016;30:2114.

11. Perez LM, Naidu SI, Joseph DB. Outcome and cost analysis of operative versus nonoperative management of neonatal multicystic dysplastic kidneys. J Urol 1998;160:1207.

12. Cambio AJ, Evans CP, Kurzrock EA. Non-surgical management of multicystic dysplastic kidney. BJU Int 2008;101.

13. Bowlin PR, Farhat WA. Laparoscopic nephrectomy and partial nephrectomy: intraperitoneal, retroperitoneal, single site. Urol Clin North Am 2015;42:31.

14. Gonzales Leon T. Laparoscopic nephrectomy: different techniques and approaches. Curr Urol Rep 2015;15:7.

15. Kim C, McKay K, Docimo SD. Laparoscopic nephrectomy in children: systematic review of transperitoneal and retroperitoneal approaches. Urology 2009;73:280.

16. McDougall EM, Clayman RV. Laparoscopic nephrectomy for benign disease: comparison of the transperitoneal and retroperitoneal approaches. J Endourol 1996:10:45.

17. Mattioli G, Pini Prato A, Costanzo S. Nephrectomy for multicystic dysplastic kidney and renal hypodysplasia in children: where do we stand? Pediatr Surg Int 2010;26:523. 18. Borzi PA. A comparison of the lateral and posterior retroperitoneo- scopic approachfoe complete and partial nephroureterectomy in children. BUJ Int 2001;87:517.

19. Urbanowics W, Wieczolek M, Suliskaswski A. Retroperitoneoscopic nephrectomy in the prone position in children (point of technique). Eur Urol 2002;42:516-9.

20. Goyal A, Hennayeke S. Prone retroperitoneoscopic approach for heminephrectomy: Specific advantages relating to access to vascular pedicle. J Pediatr Urol 2010;6:153-6.

21. You D, Hong S, Lee C, Kim RS. Feasibility and safety of laparoscopic ablative renal surgery in infants: comparative study with children. J Urol 2012;188:1330.

22. Esposito C, Lima M, Mattioli G, et al. Complications od paediatric urological laparoscopi: mistakes and risks. J Urol 2003;169:1490.

23. Esposito C, Mattioli G, Monguzzi GL, et al. Complications and conversions of pediatric videosurgery: the Italian multicentric experience on 1689 procedures. Surg Endosc 2002;5:795.

24. Varlet F, Petit T, Leclair MD, et al. Laparoscopic treatment of renal cancer in children: a multicentric study and review of oncological and surgical complications. J Pediatr Urol 2014;10:500.

25. Duarte RJ, Cristofani LM, Filho VO, et al. Videolaparoscopic radical nephrectomy after chemotherapy I the treatment of Wilms' tumor: long term results of a pioneer group. J Pediatr Urol 2017;13:50.

26. Torino G, Innocenzi M, Mele E, et al. Comparison of native ureteral ligation and open nephrectomy for pediatric renal transplantation. J Urol 2016;196:875.

27. Janetschek G, Seibold J Dadmayr C, et al. Laparoscopic heminephroureterectomy in pediatric patients. J Urol 1997;158: 1928.

28. El-Ghoneimi A, Valla JS, Steyaert H, et al. Laparoscopic renal surgery via a retroperitoneal approach in childeen. J Urol 1998; 160:1138-41.

29. Lee RS, Retik AB, Borer JG, et al. Pediatric retroperitoneal laparoscopic partial nephrectomy: comparison with an age matched cohort of open surgery. J Urol 2005;174:708-11.

30. Esposito C, Varlet F, Patkowski D, et al. Laparoscopic partial nephrectomy in duplex kidneys I infants and children: results of an European multicentric survey. Surg Endosc 2015; 29:3469.

31. Castellan M, Gosalbez R, Carmack AJ, et al. Transperitoneal and retropeitoneal laparoscopic heminephrectomy: what approach for which patient? J Urol 2006;176:2636.

32. Leclair MD, Vidal I, Suply E, et al. Retroperitoneal laparoscopic heminephrectomy in duplex kidney in infants and children: a 15-year experience. Eur Urol 2009;56:385-9.

33. Marszalek M, Chromecki T, Al-Ali BM, et al. Laparoscopic partialnephrectomy: a marche -pair comparison of the transperitoneal versus the retroperitoneal approach. Urology 2011;77:109-13.

34. Cabezali D, Maruszewski P, Lopez F, et al. complicatios and late outcomein transperitoneal laparoscopic heminephrectomy for duplex kidney in children. J Endourol 2013;27:133-8.

35. Esposito C, Escolino M, Castagnetto M, et al. Retroperitoneal and laparoscopic heminehrectomy in duplex kidney in inats and children. Transl Pediatr 2016;5:245-50.

36. Tejwani R, Young BJ, Wang HS, et al. Open versus minimally invasive surgical approaches in pediatric urology: trends I utilization and complications. J Pediatr Urol 2017;13:e1-e9.

37. Tejwani R, Wang HS, Young BJ, et al. Increased pediatric subspecialization is associated with decreased surgical complication ratesfor inpatient pediatric urology procedures. J Pediatr Urol 2016;12:e1-7. 